\title{
Concurrent chemoradiation therapy tailored to the older adults with esophageal cancer: state of the art and the future
}

This article was published in the following Dove Press journal:

Clinical Interventions in Aging

\section{Tao Song' \\ Min Fang' \\ Shixiu $\mathrm{Wu}^{2}$}

'Department of Radiation Oncology, Zhejiang Provincial People's Hospital, People's Hospital of Hangzhou Medical College, Hangzhou 310000,

Zhejiang, People's Republic of China; ${ }^{2}$ Department of Radiation Oncology, Hangzhou Cancer Hospital, Hangzhou 310000, Zhejiang, People's Republic of China
Correspondence: Shixiu Wu Department of Radiation Oncology, Hangzhou Cancer Hospital, No 34, Yanguan Lane, Shangcheng District, Hangzhou 310000, People's Republic of China $\mathrm{Tel}+8657186826086$ Fax +86 57I 8606228 I Email wushixiu@medmail.com.cn
Purpose: The aim of this study was to review the published literature addressing the question of whether geriatric assessment (GA) should be routinely applied in the treatment of older adults with esophageal cancer (EC) who have received definitive concurrent chemoradiotherapy (dCRT).

Materials and methods: A literature search of PubMed, Embase, and Cochrane Library was performed. Studies that contained original data outlining the inclusion and exclusion criteria, treatment compliance rate, and severe toxicity reports were reviewed. Additionally, criteria from ongoing clinical trials in the World Health Organization and National Institutes of Health registries were reviewed to evaluate the utilization of GA-related domains in elderly EC patients who received dCRT.

Results: Twenty-nine studies were identified based on the selection criteria: five were singlearm prospective studies, and the other studies were retrospective studies. All studies used chronological age and performance status as basic descriptors for this subpopulation. The comorbidity index and the malnutrition level were mentioned in several studies. However, factors such as "Demographic data and social support," "Psychology," "Polypharmacy," and "Geriatric syndromes" were not described in any of the included studies. Unfortunately, the results were similar for the registered clinical trials. Finally, treatment compliance and toxicity profile were found to be acceptable in selected elderly EC patients.

Conclusion: The current experience for older adults with EC receiving dCRT is mainly based on the results of a series of retrospective studies. Ongoing clinical trials should routinely consider GA-related domains to select appropriate treatments for patients in the future.

Keywords: older adults, esophageal cancer, chemoradiotherapy, inclusion criteria, toxicity, geriatric assessment

\section{Introduction}

Esophageal cancer (EC) is a prevalent disease with a very poor prognosis. It is the fourth most common cause of cancer death in People's Republic of China. ${ }^{1,2}$ As life expectancy is expected to increase over time, the number of older adults with EC is likely to increase worldwide. However, due to the vast heterogeneity in the aging population, elderly patients are underrepresented in most clinical trials. How to best manage this specific population is still a great challenge. In a recently published study, 21,593 elderly (aged over 70 years) EC patients with clinical stage II or III cancer identified from the National Cancer Database were retrospectively analyzed. Demographic characteristics revealed that $37.1 \%(8,010 / 21,593)$ of patients had received definitive concurrent chemoradiotherapy (dCRT). ${ }^{3}$ Considering that some patients were diagnosed with clinical stage I or IVa cancer, we believe that more than $37.1 \%$ 
of elderly EC patients were recommended dCRT as the firstline of cancer therapy.

Currently, there is consensus within the geriatric oncology community that chronological age alone is a poor descriptor of the heterogeneity that exists within the aging process. Variation in physiological reserves, comorbidities, geriatric syndromes, and limited social support networks also contribute to the risk associated with aggressive treatment modalities. In light of these circumstances, the International Society of Geriatric Oncology (SIOG) and National Comprehensive Cancer Network established recommendations for geriatric assessment (GA) to help oncologists determine suitable treatment choices for older patients. ${ }^{4-6}$ However, the role of GA in the application of dCRT for elderly EC patients remains to be clarified.

Thus, the purpose of this study was to conduct a systemic review of all existing observational cohort studies on the use of GA in the treatment decisions for elderly EC patients receiving dCRT. However, due to the low number of previous studies that used GA in pretreatment evaluation, we summarized the inclusion and exclusion criteria and other important aspects related to GA, including treatment compliance and toxicity for older adults with EC, focusing on whether GA-related domains were routinely applied. We provide some potential suggestions for registered clinical trials in the future.

\section{Materials and methods}

\section{Search strategy and article selection}

A systematic literature search for articles published between January 1, 1990 and December 31, 2017 was conducted in PubMed, Embase, and Cochrane Library with the assistance of a research librarian. The primary outcome was to identify all publications containing original data on the use of dCRT in older adults with EC, including study inclusion and exclusion criteria, treatment compliance, and reports of severe toxicity. The following terms were used as search terms: (esophageal or esophagus or oesophageal) and (cancer or carcinoma or neoplasm or tumor or tumour) and (aged or older or elderly) and concurrent and treatment. Exclusion criteria for search results were as follows: the study involved neoadjuvant chemoradiotherapy (nCRT) with surgery; the study was in Phase I; the study involved palliative chemoradiotherapy; the study was not published with the full text; the study was combined with nCRT or radiotherapy alone; and the study results were not reported exactly. The search did not restrict the type of publication but was limited to English language.

To identify ongoing clinical trials focusing on the efficiency and safety of dCRT for older adults with EC, the World
Health Organization registry network (http://www.who.int/ ictrp/network/primary) and the National Institutes of Health (NIH) clinical trial registry (http://www.ClinicalTrials.gov) were searched on December 31, 2017, using the search terms "esophageal cancer" and "radiotherapy." The search was limited to only interventional trials currently recruiting elderly EC patients or intending to start recruitment.

\section{Review methods}

The abstract of each article was first reviewed by the corresponding author (SW). Irrelevant citations were removed according to the selection criteria, thereby creating a preliminary set of potentially relevant publications. Then, the fulltext articles were distributed to the review team along with an evaluation form customized for reviewing the GA-related domains previously mentioned: study selection criteria, reports of severe toxicity and treatment compliance (TS and MF). The evaluation results were compared and reevaluated until consensus was reached between the two reviewers. For ongoing clinical trials, the following data were also extracted from the registry website: registration number, country, chemotherapy regimen, inclusion age, and GA-related domains mentioned in the selection criteria.

To allow the combination of performance status data, a score of 100 on the Karnofsky Performance Scale Index (KPS) was considered equivalent to an Eastern Cooperative Oncology Group performance status (ECOG PS) scale score of 0 ; a score of $80-90$ on the KPS was considered equivalent to an ECOG PS score of 1; and a score of 60-70 on the KPS scale was considered equivalent to an ECOG PS score of 2.7 GA-related domains were classified using the following categories: "demographic data and social support," "comorbidity," "functional status," "cognition," "psychology," "nutrition," "polypharmacy," and "geriatric syndromes," following the SIOG recommendations released in $2014 .^{5}$ In the published reports, restrictions in selection criteria were labeled as "specific criterion mentioned in the report" or "not limited" or "not reported" for each category listed above. In the registered clinical trials, restrictions in selection criteria were labeled as "strict," "mentioned," or "not reported" for GA-related domains.

\section{Results \\ Description of studies and methodological issues}

After examination of the relevant abstracts and full-text articles by the review group, a total of 29 published studies involving 1,487 EC patients were incorporated into the systematic review. A flow diagram of citation retrieval and 
selection is presented in Figure 1. The basic characteristics of patients and GA-related domains mentioned in the methods and results are summarized in Table $1 .{ }^{8-36}$ Of these 29 reports, 5 studies were single-arm prospective clinical trials and the other studies were retrospective studies. Of the five prospective studies, one study used radiation with concurrent targeted therapy of gefitinib. Patient sample size in the eligible studies ranged from 7 to 188 . Nearly $84.4 \%(1,255 / 1,487)$ of patients were diagnosed with esophageal squamous cell carcinoma (ESCC). Eleven reports defined the chronological age of 70 as the cutoff age for older adults. Among studies, considerable variation was found across chemotherapy regimens, radiation therapy doses, and radiotherapy parameters. These methodological issues made comparing the results challenging both within study groups and between studies.

\section{GA-related issues}

Unfortunately, "demographic data and social support," "psychology," "polypharmacy," and "geriatric syndromes" categories were not described in any of the selected studies (Table S1). Only one prospective study conducted by Servagi-Vernat et $\mathrm{al}^{8}$ used Folstein's test as the GA for "Cognition." The other domains are presented in the following.

\section{Functional status}

ECOG PS and KPS were the two most commonly used tools of functional status assessment in daily oncological practice. Among the 29 selected studies, 2 studies did not clearly report the functional status of elderly patients. For the remaining 27 studies, 15 studies used the basic ECOG PS score of 0-2 for inclusion, and 6 studies restricted the ECOG PS score to 0-1 for inclusion. Among the 16 studies that clearly demonstrated the proportion of performance status scores across patients, $77.2 \%(586 / 759)$ of the patients were assessed at an ECOG PS score of $0-1$, and 173 patients were assessed at an ECOG PS score of 2-3. It is worth mentioning that only one prospective study, also conducted by Servagi-Vernat et al, ${ }^{8}$ used Instrumental Activity Of Daily Living as an additional instrument for evaluating functional status.

\section{Comorbidity}

Comorbidity is a characterization factor for elderly cancer patients. In this review, 18 studies mentioned comorbidities in enrolled patients. Among them, the Charlson Comorbidity Index (CCI), or its age-adjusted version (ACCI), was the most common tool for assessing comorbidity. In the five prospective studies, two clinical trials, both conducted by Servagi-Vernat et al, ${ }^{8}$ used inclusion criteria of CCI

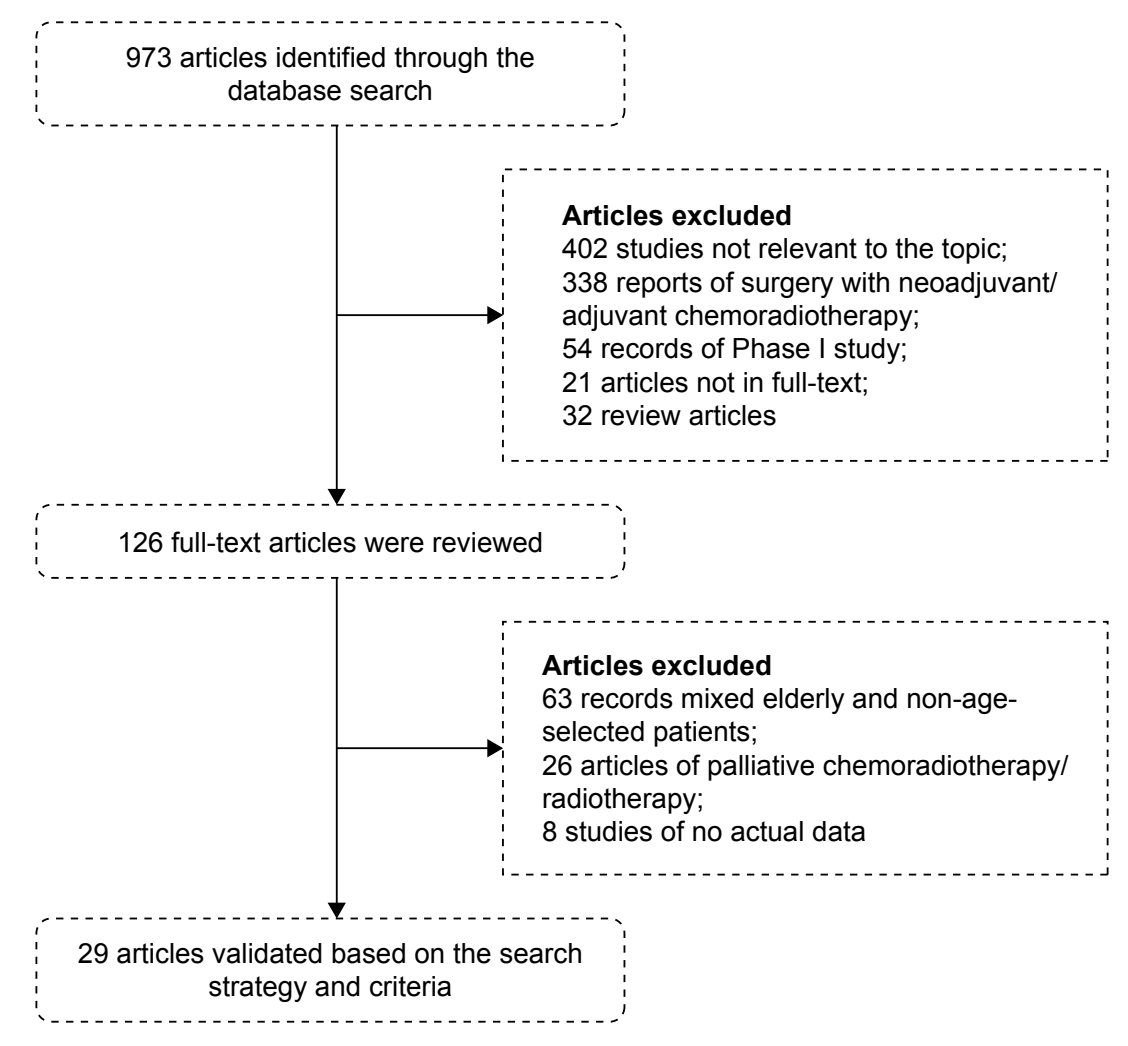

Figure I Schematic presentation of methodology used and selection criteria.

Note: Search and selection criteria conducted in accordance with PRISMA statement criteria.

Abbreviation: PRISMA, Preferred Reporting Items for Systematic Reviews and Meta-Analyses. 


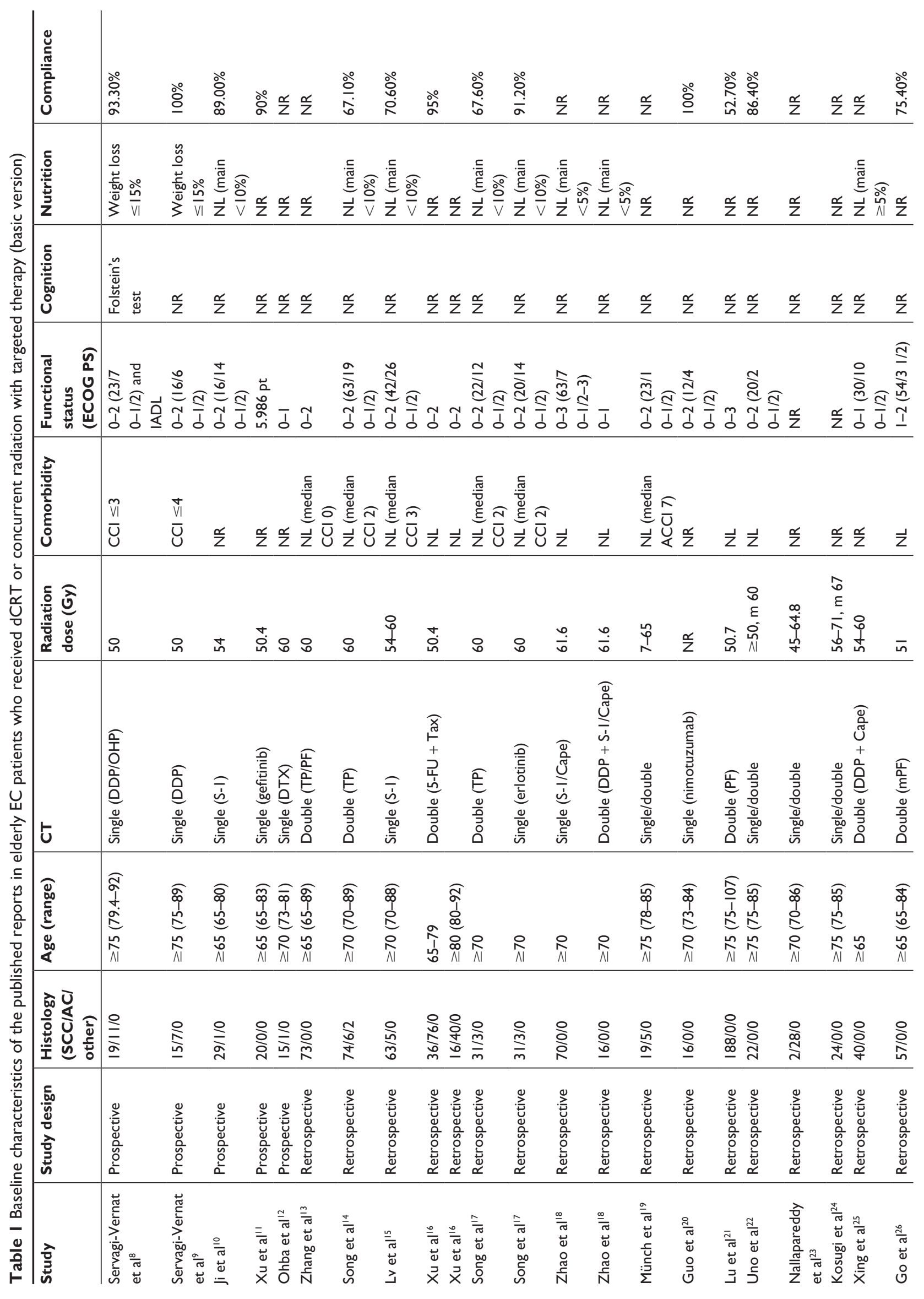




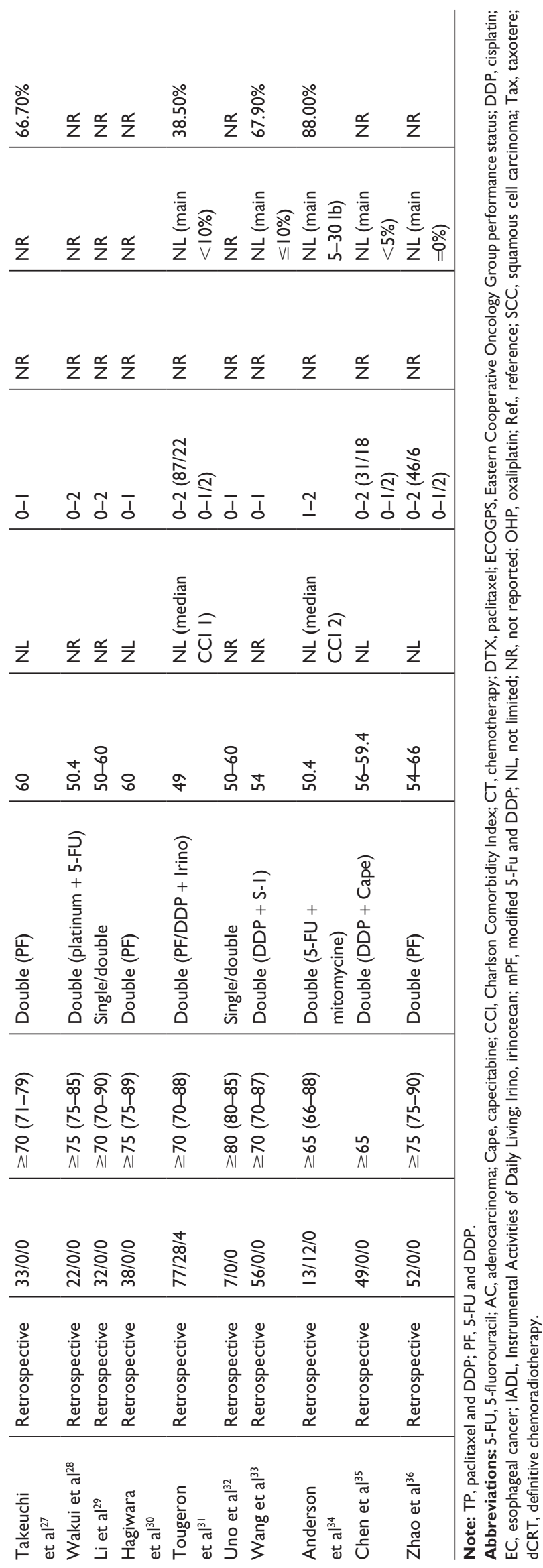

scores $<3$ and $<4$. Among the 24 retrospective studies, 8 studies did not mention any information regarding the comorbidities of their enrolled patients. On the contrary, seven retrospective reports indicated the median CCI/ACCI clearly, with three publications reporting a median CCI of 2 .

\section{Nutrition}

Malnutrition would certainly increase the risk of antitumor therapy and influence clinical outcomes, especially for elderly EC patients. In our systematic review, we found that the two prospective trials by Servagi-Vernat et a ${ }^{8}$ had criteria excluding patients with weight loss of more than $15 \%$ from baseline. In another Phase II trial conducted by Ji et al, ${ }^{10}$ weight loss was not used as an exclusion criterion, but over half of the patients $(17 / 30,56.7 \%)$ had $<10 \%$ weight loss over nearly 6 months. Fourteen of the 24 retrospective studies did not reveal any results related to nutrition. The remaining 10 retrospective studies did not restrict the inclusion criteria in terms of malnutrition, with the main proportion of weight loss ranging from $0 \%$ to $<10 \%$.

\section{Treatment compliance and severe toxicity} Different chemoradiation therapies could result in variable treatment compliance. In our systematic review, a total of 16 studies detailed the wide range of treatment compliance in elderly EC patients. The compliance rates ranged from $38.5 \%$ to $100 \%$. Single-agent chemotherapy or targeted therapy had relatively higher treatment compliance rates $(70.6 \%-100.0 \%)$ than did double-agent chemotherapies $(38.5 \%-95.0 \%)$. The results of grade 3 or higher acute treatment-related toxicities are also presented in Table S2. Leukopenia was the most common acute toxicity reported in the literature, with incidences ranging from $0 \%$ to $72.7 \%$. Ten studies also reported the incidence of severe neutropenia, ranging from $0 \%$ to $53.6 \%$. The incidences of two other hematologic toxicities, anemia, and thrombocytopenia were $0 \%-45.5 \%$ and $0 \%-50.0 \%$, respectively. Esophagitis was the most common radiation-related acute toxicity for elderly EC patients, ranging from $0 \%$ to $39.3 \%$. Nausea/vomiting was the second most common during the acute phase. Other acute toxicities (grade $>3$ ) were rare with moderate tolerance.

\section{Registered clinical trials}

Nine registered clinical trials were found in the WHO and NIH registry network, based on our research methods (Table 2). Among these ongoing studies, six trials distributed across the mainland People's Republic of China. Trial NCT 02735057, conducted in France, was the only study that 


\begin{tabular}{|c|c|c|c|c|c|c|c|c|c|c|c|}
\hline$\frac{\Delta}{z}$ & & & & & & & & 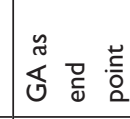 & & 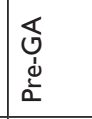 & 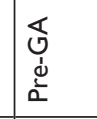 \\
\hline 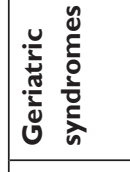 & $\frac{x}{z}$ & $\frac{\alpha}{z}$ & $\frac{\alpha}{z}$ & $\frac{\alpha}{z}$ & $\frac{x}{z}$ & $\frac{\alpha}{z}$ & $\frac{\tilde{\alpha}}{z}$ & $\frac{\alpha}{z}$ & $\frac{\tilde{z}}{z}$ & $\frac{\approx}{z}$ & $\frac{\alpha}{z}$ \\
\hline 产 & $\Sigma$ & $\Sigma$ & $\Sigma$ & $\frac{\alpha}{z}$ & $\frac{\infty}{z}$ & $\frac{x}{z}$ & $\frac{x}{z}$ & $\frac{\alpha}{z}$ & $\frac{x}{z}$ & $\frac{\alpha}{z}$ & $\frac{\alpha}{z}$ \\
\hline 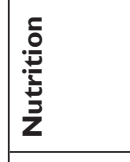 & 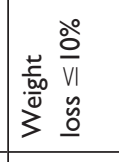 & $\frac{\alpha}{z}$ & $\frac{\alpha}{z}$ & $\frac{\alpha}{z}$ & $\frac{\alpha}{z}$ & $\frac{\alpha}{z}$ & $\frac{\alpha}{z}$ & $\frac{\alpha}{z}$ & 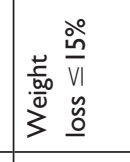 & 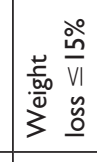 & 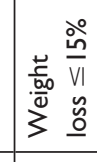 \\
\hline 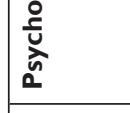 & $\frac{\alpha}{z}$ & $\Sigma$ & $\Sigma$ & $\Sigma$ & $\frac{\alpha}{z}$ & $\frac{\alpha}{z}$ & $\frac{\alpha}{z}$ & $\frac{\alpha}{z}$ & $\Sigma$ & $\Sigma$ & $\Sigma$ \\
\hline نั & $\Sigma$ & $\frac{\alpha}{z}$ & $\frac{\alpha}{z}$ & $\frac{\alpha}{z}$ & $\frac{x}{z}$ & $\frac{\alpha}{z}$ & $\Sigma$ & $\frac{x}{z}$ & $\Sigma$ & $\Sigma$ & $\Sigma$ \\
\hline 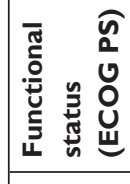 & & ટે & $\bar{d}$ & $\bar{\delta}$ & $\bar{\delta}$ & $\bar{\delta}$ & s. & sิ & $\hat{\delta}$ & ș & ภิ \\
\hline 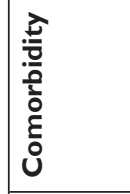 & 蒿 & 䓂 & 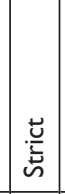 & $\Sigma$ & 蒿 & 菩 & 蒡 & 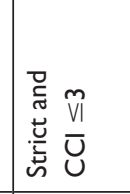 & 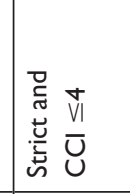 & 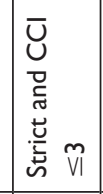 & 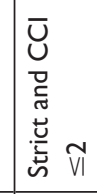 \\
\hline 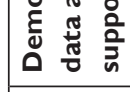 & $\frac{x}{z}$ & $\frac{x}{z}$ & $\frac{x}{z}$ & $\frac{x}{z}$ & $\frac{\tilde{z}}{z}$ & $\frac{x}{z}$ & zz & $\frac{\tilde{z}}{z}$ & $\frac{\alpha}{z}$ & & $\Sigma$ \\
\hline$\underset{4}{\$ 0}$ & 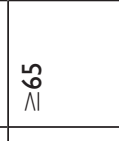 & 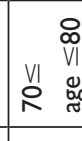 & 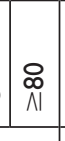 & 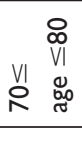 & $\underset{N}{8}$ & 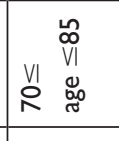 & 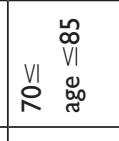 & $i_{1}^{2}$ & 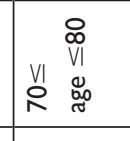 & & 离 \\
\hline 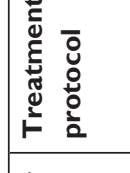 & 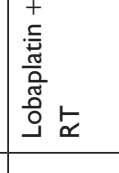 & $\begin{array}{l}\frac{b}{a} \\
+ \\
x \\
a \\
\end{array}$ & & 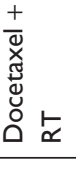 & 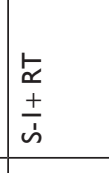 & 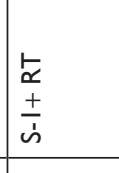 & 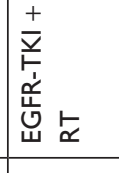 & \begin{tabular}{|l}
$\underline{a}$ \\
$\frac{ \pm}{\Delta}$ \\
\end{tabular} & 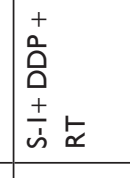 & 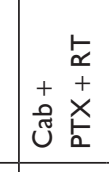 & \\
\hline نَ & 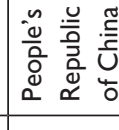 & ᄃ & & 总 & 总 & 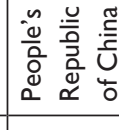 & 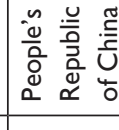 & 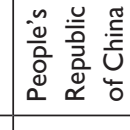 & 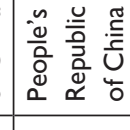 & 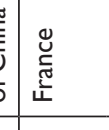 & \\
\hline$\underline{\underline{a}}$ & 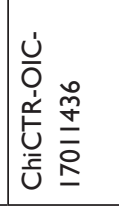 & 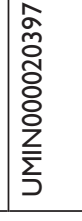 & & 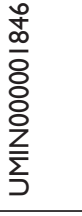 & 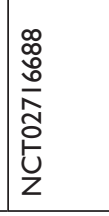 & 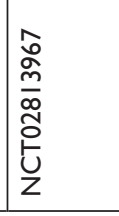 & 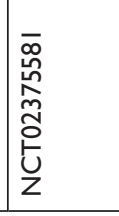 & 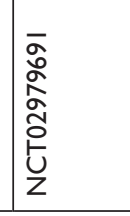 & 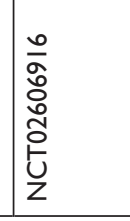 & 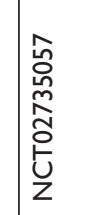 & \\
\hline
\end{tabular}


detailed their study protocol in another journal. ${ }^{37}$ Among the nine studies, chronological age, comorbidity, and functional status were commonly established as notable markers for the assessment of the elderly. To our surprise, the inclusion criteria of malnutrition was only strictly restricted in three studies. Other meaningful markers such as "cognitive status," "polypharmacy," and "geriatric syndromes" were sporadically mentioned in these studies.

\section{Discussion}

Currently, high quality data from older adults with EC treated with dCRT are extremely limited, according to our systematic review. In the largest retrospective study to date, involving patients over 70 years of age diagnosed with esophageal cancer, Vlacich et $\mathrm{al}^{3}$ showed that over $37.1 \%$ of elderly patients received dCRT and had a satisfactory median overall survival (OS) time of 14.0 months (95\% CI: 13.5-14.5). ${ }^{3}$ By contrast, for elderly patients treated with trimodality therapy, the median OS time was only increased to 19.3 months (95\% CI: 17.1-21.5) with the added sacrifice of abnormal esophageal function that had an equally important impact on psychological and geriatric syndromes. Using multivariate logistic regression, they found that age over 80 years (odds ratio: 0.79 ) was a strong indicator of decreased OS likelihood and that patients with clinical stage III, tumors in the upper and middle third of the esophagus, a Charlson-Deyo comorbidity score $<1$, adenocarcinoma histology, and being treated at a non-academic cancer center were more likely to receive esophagectomy in the USA. Another large sample retrospective analysis from the National Cancer Database provided the opportunity to review treatment utilization and outcomes in patients $\geq 80$ years of age diagnosed with stage I esophageal cancer. Their analysis revealed that $22 \%$ of elderly patients received dCRT among the 923 patients. The survival curve demonstrated a remarkable benefit over observation alone (5-year OS: $20 \%$ vs $7 \%, P<0.001$ ) with the acceptable toxicity profile of dCRT. ${ }^{38}$

Apart from known factors that are often associated with treatment disparities such as socioeconomic status and chronologic age, the functional and nutritional status at diagnosis influenced treatment utilization. In 2010, Tougeron et $\mathrm{al}^{39}$ investigated the baseline parameters that influenced both therapeutic decisions and outcomes in 282 elderly EC patients. Their outcomes further suggested that elderly EC patients with good performance status, good nutritional status, and without serious comorbidities were able to benefit from curative treatment without severe adverse events. ${ }^{39}$ In addition to functional and nutritional status for the elderly patients, items of psychology and polypharmacy also play an important role in treatment decisions. In a randomized controlled study evaluating the psychological nursing interventions on personality characteristics and quality of life of EC patients, Cheng et $\mathrm{al}^{40}$ proved that a psychological nursing intervention could affect the personality characteristics of EC patients and improve quality of life. Furthermore, analyses from elderly Danish cancer patients (including EC patients) showed that newly diagnosed elderly cancer patients ( $\geq 70$ years) use more drugs than control patients (odds ratio: 1.76). Drug use increased markedly in the last 6 months prior to cancer diagnosis. ${ }^{41}$ This finding suggested an increased symptom burden in elderly patients and might serve as a warning signal for general practitioners.

In general, elderly EC patients were at increased risk of severe treatment-related toxicities and had a lower incidence of treatment compliance than younger patients. Based on the current review, compliance rates ranged from $38.5 \%$ to $100 \%$. Single-agent chemotherapy or targeted therapy seemed to have higher treatment compliance $(70.6 \%-100.0 \%)$ than did double-agent chemotherapies (38.5\%-95.0\%). Unlike combined therapies for non-age-selected patients, our recent Phase III trial demonstrated that dCRT (TP regimen and radiation therapy) with erlotinib could achieve significantly better OS and locoregional control than standard dCRT in patients with inoperable ESCC. Furthermore, this trial confirmed that, aside from rash and radiation esophagitis, the incidence of grade 3 or greater toxicities were manageable and did not differ across the four groups. ${ }^{42}$ Traditionally, it was thought that elderly patients have fewer body function reserves, shorter life expectancy, and more need for socioeconomic support than young patients. Therefore, this finding suggests that aggressive treatment decisions might not be suitable for this unique and challenging subpopulation. In a small-sample retrospective analysis, Wang et $\mathrm{al}^{33}$ observed a significantly higher incidence of severe leukocytopenia in ESCC patients aged $\geq 75$ than in patients aged between 70 and 75 years $(41.9 \%$ vs $72.0 \%$, respectively, $P=0.024)$. Hence, a detailed and full pretreatment evaluation, especially containing GA-related domains should be routinely applied to select appropriate elderly patients to receive dCRT.

\section{Conclusion}

Our systematic review fully demonstrates that the current experience of receiving dCRT in elderly patients is based on the results of a series of retrospective studies. High-level evidence of RCTs remains lacking. Ongoing prospective clinical trials should take GA-related domains into routine 
consideration to select appropriate care for patients in the future. Survival benefits and the toxicity profiles should also be balanced among these treatment strategies.

\section{Acknowledgments}

The authors thank Li Zhang, MD, and Zhao Jing, MD, for their help with translation and proofreading. This research received no specific grant from any funding agency in the public, commercial, or not-for-profit sectors.

\section{Disclosure}

The authors report no conflicts of interest in this work.

\section{References}

1. Siegel R, Ma J, Zou Z, Jemal A. Cancer statistics, 2014. CA Cancer J Clin. 2014;64(1):9-29.

2. Chen W, Zheng R, Baade PD, et al. Cancer statistics in China, 2015. CA: a cancer journal for clinicians. 2016;66(2):115-132.

3. Vlacich G, Samson PP, Perkins SM, et al. Treatment utilization and outcomes in elderly patients with locally advanced esophageal carcinoma: a review of the National Cancer Database. Cancer Med. 2017; 6(12):2886-2896.

4. Extermann M, Aapro M, Bernabei R, et al; Task Force on CGA of the International Society of Geriatric Oncology. Use of comprehensive geriatric assessment in older cancer patients: recommendations from the task force on CGA of the International Society of Geriatric Oncology (SIOG). Crit Rev Oncol Hematol. 2005;55(3):241-252.

5. Wildiers H, Heeren P, Puts M, et al. International Society of Geriatric Oncology consensus on geriatric assessment in older patients with cancer. J Clin Oncol. 2014;32(24):2595-2603.

6. Won E. Issues in the management of esophageal cancer and geriatric patients. Chin Clin Oncol. 2017;6(5):51.

7. Hamaker ME, Stauder R, van Munster BC. Exclusion of older patients from ongoing clinical trials for hematological malignancies: an evaluation of the National Institutes of Health Clinical Trial Registry. Oncologist. 2014;19(10):1069-1075.

8. Servagi-Vernat S, Créhange G, Roullet B, et al. Phase II study of a platinum-based adapted chemotherapy regimen combined with radiotherapy in patients 75 years and older with esophageal cancer. Drugs Aging. 2015;32(6):487-493.

9. Servagi-Vernat S, Bosset M, Crehange G, et al. Feasibility of chemoradiotherapy for oesophageal cancer in elderly patients aged $\geq 75$ years: a prospective, single-arm phase II study. Drugs Aging. 2009;26(3):255-262.

10. Ji Y, Du X, Tian Y, et al. A phase II study of S-1 with concurrent radiotherapy in elderly patients with esophageal cancer. Oncotarget. 2017;8(47):83022-83029.

11. Xu Y, Zheng Y, Sun X, et al. Concurrent radiotherapy with gefitinib in elderly patients with esophageal squamous cell carcinoma: Preliminary results of a phase II study. Oncotarget. 2015;6(35):38429-38439.

12. Ohba A, Kato K, Ito Y, et al. Chemoradiation therapy with docetaxel in elderly patients with stage II/III esophageal cancer: A phase 2 trial. Adv Radiat Oncol. 2016;1(4):230-236.

13. Zhang $\mathrm{P}, \mathrm{Xi} \mathrm{M}$, Zhao $\mathrm{L}$, et al. Is there a benefit in receiving concurrent chemoradiotherapy for elderly patients with inoperable thoracic esophageal squamous cell carcinoma? PLoS One. 2014;9(8):e105270.

14. Song T, Zhang X, Fang M, Wu S. Concurrent chemoradiotherapy using paclitaxel plus cisplatin in the treatment of elderly patients with esophageal cancer. Onco Targets Ther. 2015;8:3087-3094.
15. Lv S, Fang M, Yang J, et al. Long-term results of definitive concurrent chemoradiotherapy using S-1 in the treatment of geriatric patients with esophageal cancer. Onco Targets Ther. 2016;9:5389-5397.

16. Xu C, Xi M, Moreno A, et al. Definitive Chemoradiation Therapy for Esophageal Cancer in the Elderly: Clinical Outcomes for Patients Exceeding 80 Years Old. Int J Radiat Oncol Biol Phys. 2017;98(4): 811-819.

17. Song T, Du D, Zhang X, Fang M, Wu S. Comparative study of radiotherapy plus erlotinib versus chemoradiotherapy for elderly patients with esophageal cancer: a propensity score-matched analysis. Dis Esophagus. 2017;30(9):1-10.

18. Zhao L, Zhou Y, Pan H, et al. Radiotherapy Alone or Concurrent Chemoradiation for Esophageal Squamous Cell Carcinoma in Elderly Patients. J Cancer. 2017;8(16):3242-3250.

19. Münch S, Heinrich C, Habermehl D, Oechsner M, Combs SE, Duma MN. Primary radio(chemo)therapy for esophageal cancer in elderly patients: are efficiency and toxicity comparable with younger patients? Eur $J$ Med Res. 2017;22(1):24.

20. Guo JH, Chen MQ, Chen C, Lu HJ, Xu BH. Efficacy and toxicity of nimotuzumab combined with radiotherapy in elderly patients with esophageal squamous cell carcinoma. Mol Clin Oncol. 2015;3(5): $1135-1138$.

21. Lu X, Wu H, Wang J, Xu J. Short- and long-term outcomes of definitive chemoradiotherapy in patients with esophageal carcinoma aged $\geq 75$ years. Mol Clin Oncol. 2014;2(2):297-301.

22. Uno T, Isobe K, Kawakami H. Efficacy and toxicities of concurrent chemoradiation for elderly patients with esophageal cancer. Anticancer Res. 2004;24:2483-2486.

23. Nallapareddy S, Wilding GE, Yang G, Iyer R, Javle M. Chemoradiation is a tolerable therapy for older adults with esophageal cancer. Anticancer Res. 2005;25(4):3055-3060.

24. Kosugi S, Sasamoto R, Kanda T, Matsuki A, Hatakeyama K. Retrospective review of surgery and definitive chemoradiotherapy in patients with squamous cell carcinoma of the thoracic esophagus aged 75 years or older. Jpn J Clin Oncol. 2009;39(6):360-366.

25. Xing L, Liang Y, Zhang J, et al. Definitive chemoradiotherapy with capecitabine and cisplatin for elder patients with locally advanced squamous cell esophageal cancer. J Cancer Res Clin Oncol.2014;140(5): 867-872.

26. Go SI, Sup Lee W, Hee Kang M, et al. Response to concurrent chemoradiotherapy as a prognostic marker in elderly patients with locally advanced esophageal cancer. Tumori. 2012;98(2):225-232.

27. Takeuchi S, Ohtsu A, Doi T, et al. A retrospective study of definitive chemoradiotherapy for elderly patients with esophageal cancer. $\mathrm{Am}$ J Clin Oncol. 2007;30(6):607-611.

28. Wakui R, Yamashita H, Okuma K, et al. Esophageal cancer: definitive chemoradiotherapy for elderly patients. Dis Esophagus. 2010;23(7): $572-579$.

29. Li X, Zhao LJ, Liu NB, et al. Feasibility and efficacy of concurrent chemoradiotherapy in elderly patients with esophageal squamous cell carcinoma: a respective study of 116 cases from a single institution. Asian Pac J Cancer Prev. 2015;16(4):1463-1469.

30. Hagiwara K, Kochi M, Fujii M, et al. Radiochemotherapy for esophageal squamous cell carcinoma in elderly patients. Hepatogastroenterology. 2014;61(134):1617-1622.

31. Tougeron D, Di Fiore F, Thureau S, et al. Safety and outcome of definitive chemoradiotherapy in elderly patients with oesophageal cancer. Br J Cancer. 2008;99(10):1586-1592.

32. Uno T, Kawakami H, Funami Y, et al. Chemoradiation for patients with esophageal cancer aged 80 and older. Anticancer Res. 2001; 21(6A):4095-4097.

33. Wang H, Li G, Chen L, Duan Y, Zou C, Hu C. Definitive concurrent chemoradiotherapy with S-1 and cisplatin in elderly esophageal squamous cell carcinoma patients. J Thorac Dis. 2017;9(3): 646-654. 
34. Anderson SE, Minsky BD, Bains M, Hummer A, Kelsen D, Ilson DH. Combined modality chemoradiation in elderly oesophageal cancer patients. Br J Cancer. 2007;96(12):1823-1827.

35. Chen F, Luo H, Xing L, Liang N, Xie J, Zhang J. Feasibility and efficiency of concurrent chemoradiotherapy with capecitabine and cisplatin versus radiotherapy alone for elderly patients with locally advanced esophageal squamous cell carcinoma: Experience of two centers. Thorac Cancer. 2018;9(1):59-65.

36. Zhao Q, Hu G, Xiao W, et al. Comparison of definitive chemoradiotherapy and radiotherapy alone in patients older than 75 years with locally advanced esophageal carcinoma: A retrospective cohort study. Medicine (Baltimore). 2017;96(35):e7920.

37. Servagi-Vernat S, Créhange G, Bonnetain F, Mertens C, Brain E, Bosset JF. Chemoradiation in elderly esophageal cancer patients: rationale and design of a phase I/II multicenter study (OSAGE). BMC Cancer. 2017;17(1):483.

38. Moreno AC, Verma V, Hofstetter WL, Lin SH. Patterns of Care and Treatment Outcomes of Elderly Patients With Stage I Esophageal Cancer: Analysis of the National Cancer Data Base. J Thorac Oncol. 2017;12(7):1152-1160.
39. Tougeron D, Hamidou H, Scotté M, et al. Esophageal cancer in the elderly: an analysis of the factors associated with treatment decisions and outcomes. BMC Cancer. 2010;10:5-10.

40. Cheng QM, Kong CQ, Chang SY, Wei AH. Effects of psychological nursing intervention on personality characteristics and quality of life of patients with esophageal cancer. Clin Res Hepatol Gastroenterol. 2013;37(3):283-288.

41. Jorgensen TL, Herrstedt J, Friis S, Hallas J. Polypharmacy and drug use in elderly Danish cancer patients during 1996 to 2006. J Geriatr Oncol. 2012;3(1):33-40.

42. Wu SX, Wang LH, Luo HL, et al. Randomised phase III trial of concurrent chemoradiotherapy with extended nodal irradiation and erlotinib in patients with inoperable oesophageal squamous cell cancer. Eur $J$ Cancer. 2018;93:99-107. 


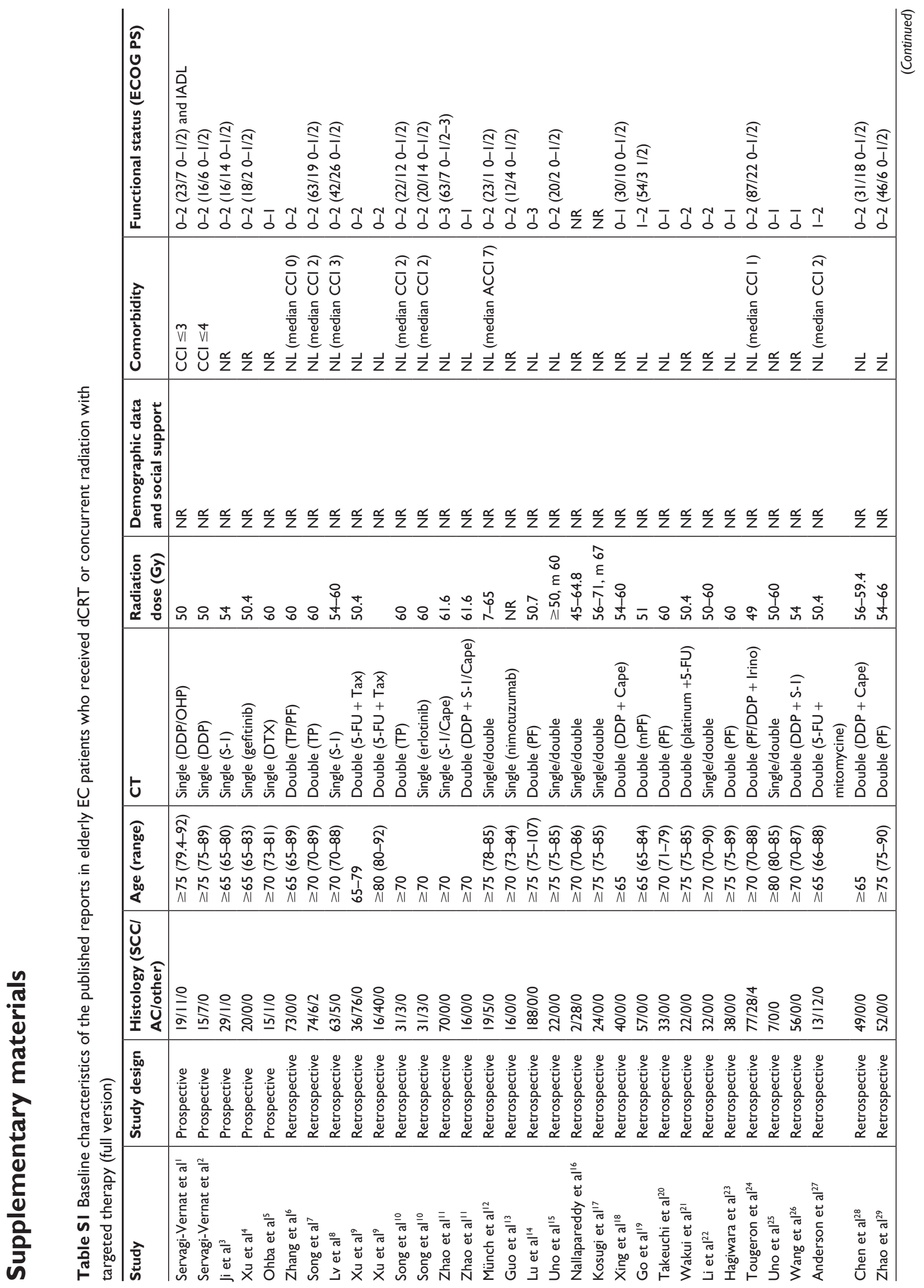




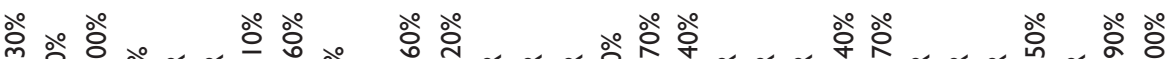

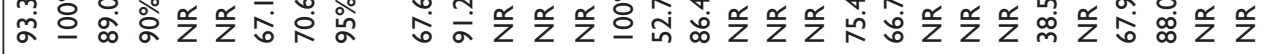

\%

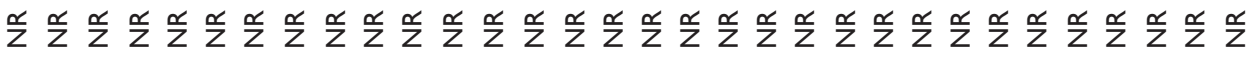

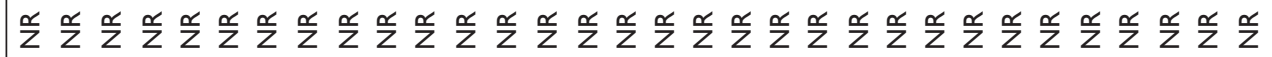

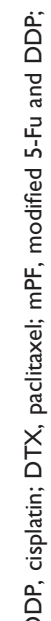

(1)

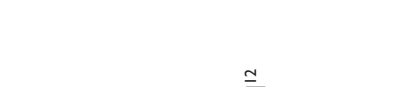




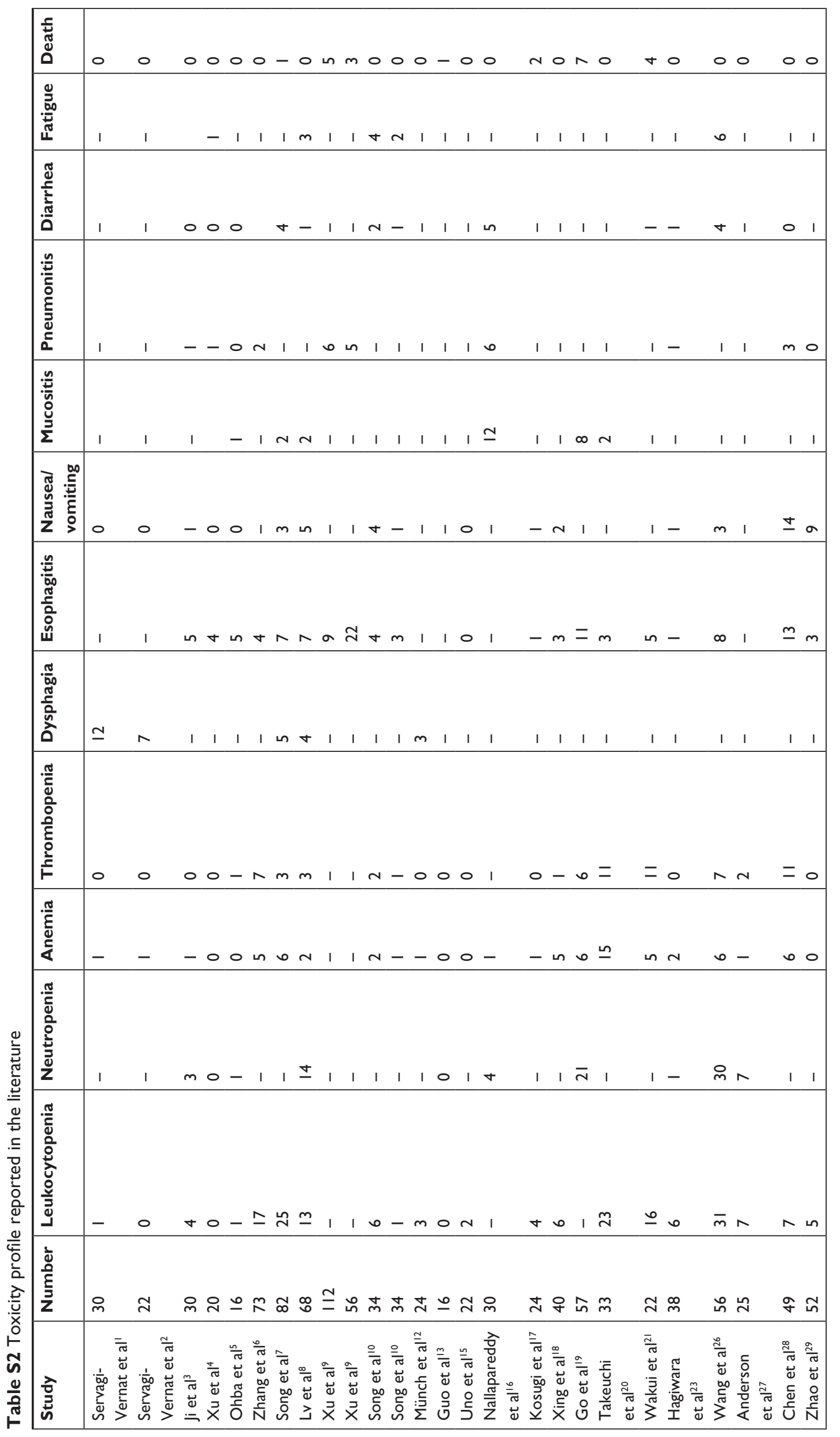




\section{References}

1. Servagi-Vernat S, Créhange G, Roullet B, et al. Phase II study of a platinumbased adapted chemotherapy regimen combined with radiotherapy in patients 75 years and older with esophageal cancer. Drugs Aging. 2015;32(6):487-493.

2. Servagi-Vernat S, Bosset M, Crehange G, et al. Feasibility of chemoradiotherapy for oesophageal cancer in elderly patients aged $\geq 75$ years: a prospective, single-arm phase II study. Drugs Aging. 2009; 26(3):255-262.

3. Ji Y, Du X, Tian Y, et al. A phase II study of S-1 with concurrent radiotherapy in elderly patients with esophageal cancer. Oncotarget. 2017; 8(47):83022-83029.

4. Xu Y, Zheng Y, Sun X, et al. Concurrent radiotherapy with gefitinib in elderly patients with esophageal squamous cell carcinoma: Preliminary results of a phase II study. Oncotarget. 2015;6(35):38429-38439.

5. Ohba A, Kato K, Ito Y, et al. Chemoradiation therapy with docetaxel in elderly patients with stage II/III esophageal cancer: A phase 2 trial. Adv Radiat Oncol. 2016;1(4):230-236.

6. Zhang $\mathrm{P}$, Xi M, Zhao L, et al. Is there a benefit in receiving concurrent chemoradiotherapy for elderly patients with inoperable thoracic esophageal squamous cell carcinoma? PLoS One. 2014;9(8):e105270.

7. Song T, Zhang X, Fang M, Wu S. Concurrent chemoradiotherapy using paclitaxel plus cisplatin in the treatment of elderly patients with esophageal cancer. Onco Targets Ther. 2015;8:3087-3094.

8. Lv S, Fang M, Yang J, et al. Long-term results of definitive concurrent chemoradiotherapy using S-1 in the treatment of geriatric patients with esophageal cancer. Onco Targets Ther. 2016;9:5389-5397.

9. Xu C, Xi M, Moreno A, et al. Definitive Chemoradiation Therapy for Esophageal Cancer in the Elderly: Clinical Outcomes for Patients Exceeding 80 Years Old. Int J Radiat Oncol Biol Phys. 2017;98(4): 811-819.

10. Song T, Du D, Zhang X, Fang M, Wu S. Comparative study of radiotherapy plus erlotinib versus chemoradiotherapy for elderly patients with esophageal cancer: a propensity score-matched analysis. Dis Esophagus. 2017;30(9):1-10.

11. Zhao L, Zhou Y, Pan H, et al. Radiotherapy Alone or Concurrent Chemoradiation for Esophageal Squamous Cell Carcinoma in Elderly Patients. J Cancer. 2017;8(16):3242-3250.

12. Münch S, Heinrich C, Habermehl D, Oechsner M, Combs SE, Duma MN. Primary radio(chemo)therapy for esophageal cancer in elderly patients: are efficiency and toxicity comparable with younger patients? Eur $J$ Med Res. 2017;22(1):24.

13. Guo JH, Chen MQ, Chen C, Lu HJ, Xu BH. Efficacy and toxicity of nimotuzumab combined with radiotherapy in elderly patients with esophageal squamous cell carcinoma. Mol Clin Oncol. 2015;3(5): 1135-1138.

14. Lu X, Wu H, Wang J, Xu J. Short- and long-term outcomes of definitive chemoradiotherapy in patients with esophageal carcinoma aged $\geq 75$ years. Mol Clin Oncol. 2014;2(2):297-301.

15. Uno T, Isobe K, Kawakami H. Efficacy and toxicities of concurrent chemoradiation for elderly patients with esophageal cancer. Anticancer Res. 2004;24:2483-2486.

Clinical Interventions in Aging

\section{Publish your work in this journal}

Clinical Interventions in Aging is an international, peer-reviewed journal focusing on evidence-based reports on the value or lack thereof of treatments intended to prevent or delay the onset of maladaptive correlates of aging in human beings. This journal is indexed on PubMed Central, MedLine,
16. Nallapareddy S, Wilding GE, Yang G, Iyer R, Javle M. Chemoradiation is a tolerable therapy for older adults with esophageal cancer. Anticancer Res. 2005;25(4):3055-3060.

17. Kosugi S, Sasamoto R, Kanda T, Matsuki A, Hatakeyama K. Retrospective review of surgery and definitive chemoradiotherapy in patients with squamous cell carcinoma of the thoracic esophagus aged 75 years or older. Jpn J Clin Oncol. 2009;39(6):360-366.

18. Xing L, Liang Y, Zhang J, et al. Definitive chemoradiotherapy with capecitabine and cisplatin for elder patients with locally advanced squamous cell esophageal cancer. J Cancer Res Clin Oncol. 2014;140(5): 867-872.

19. Go SI, Sup Lee W, Hee Kang M, et al. Response to concurrent chemoradiotherapy as a prognostic marker in elderly patients with locally advanced esophageal cancer. Tumori. 2012;98(2):225-232.

20. Takeuchi S, Ohtsu A, Doi T, et al. A retrospective study of definitive chemoradiotherapy for elderly patients with esophageal cancer. Am J Clin Oncol. 2007;30(6):607-611.

21. Wakui R, Yamashita H, Okuma K, et al. Esophageal cancer: definitive chemoradiotherapy for elderly patients. Dis Esophagus. 2010;23(7): 572-579.

22. Li X, Zhao LJ, Liu NB, et al. Feasibility and efficacy of concurrent chemoradiotherapy in elderly patients with esophageal squamous cell carcinoma: a respective study of 116 cases from a single institution. Asian Pac J Cancer Prev. 2015;16(4):1463-1469.

23. Hagiwara K, Kochi M, Fujii M, et al. Radiochemotherapy for esophageal squamous cell carcinoma in elderly patients. Hepatogastroenterology. 2014;61(134):1617-1622.

24. Tougeron D, Di Fiore F, Thureau S, et al. Safety and outcome of definitive chemoradiotherapy in elderly patients with oesophageal cancer. $\mathrm{Br}$ J Cancer. 2008;99(10):1586-1592.

25. Uno T, Kawakami H, Funami Y, et al. Chemoradiation for patients with esophageal cancer aged 80 and older. Anticancer Res. 2001; 21(6A):4095-4097.

26. Wang H, Li G, Chen L, Duan Y, Zou C, Hu C. Definitive concurrent chemoradiotherapy with $\mathrm{S}-1$ and cisplatin in elderly esophageal squamous cell carcinoma patients. J Thorac Dis. 2017;9(3):646-654.

27. Anderson SE, Minsky BD, Bains M, Hummer A, Kelsen D, Ilson DH. Combined modality chemoradiation in elderly oesophageal cancer patients. Br J Cancer. 2007;96(12):1823-1827.

28. Chen F, Luo H, Xing L, Liang N, Xie J, Zhang J. Feasibility and efficiency of concurrent chemoradiotherapy with capecitabine and cisplatin versus radiotherapy alone for elderly patients with locally advanced esophageal squamous cell carcinoma: Experience of two centers. Thorac Cancer. 2018;9(1):59-65.

29. Zhao Q, Hu G, Xiao W, et al. Comparison of definitive chemoradiotherapy and radiotherapy alone in patients older than 75 years with locally advanced esophageal carcinoma: A retrospective cohort study. Medicine (Baltimore). 2017;96(35):e7920.

CAS, Scopus and the Elsevier Bibliographic databases. The manuscript management system is completely online and includes a very quick and fair peer-review system, which is all easy to use. Visit http://www.dovepress. com/testimonials.php to read real quotes from published authors.

\section{Dovepress}

